\title{
TSUNAMI PHASE SPEED REDUCTION DUE TO WATER COMPRESSIBILTY
}

\author{
Ali Abdolali, National Oceanic and Atmospheric Administration, University Corporation for Atmospheric Research, \\ ali.abdolali@noaa.gov, abdolali@ucar.edu \\ James T. Kirby, University of Delaware, kirby@udel.edu
}

\section{INTRODUCTION}

Most existing tsunami propagation models consider the ocean to be an incompressible, homogenous medium. Recently, it has been shown that a number of physical features can slow the propagation speed of tsunami waves, including wave frequency dispersion, ocean bottom elasticity, water compressibility and thermal or salinity stratification. These physical effects are secondary to the leading order, shallow water or long wave behavior, but still play a quantifiable role in tsunami arrival time, especially at far distant locations. In this work, we have performed analytical and numerical investigations and have shown that consideration of those effects can actually improve the prediction of arrival time at distant stations, compared to incompressible forms of wave equations. We derive a modified Mild Slope Equation for Weakly Compressible fluid following the method proposed by Sammarco et al. (2013) and Abdolali et al. (2015) using linearized wave theory, and then describe comparable extensions to the Boussinesq model of Kirby et al. (2013). Both models account for water compressibility and compression of static water column to simulate tsunami waves. The mild slope model is formulated in plane Cartesian coordinates and is thus limited to medium propagation distances, while the Boussinesq model is formulated in spherical polar coordinates and is suitable for ocean scale simulations.

\section{MILD SLOPE EQUATION}

We have used a numerical model based on the solution of a hyperbolic mild slope equation, valid in weakly compressible fluids, detailed in Abdolali and Kirby (2017):

$$
\begin{aligned}
& {\left[\left(\frac{I_{1}^{n}}{c^{2}}+\frac{1}{g}\right) \tilde{\phi}_{n, t}\right]_{, t}-\nabla_{h} \cdot\left(I_{1}^{n} \nabla_{h} \tilde{\phi}_{n}\right)+\left[I_{2}^{n}+\left(\frac{\gamma}{2}\right)^{2} I_{1}^{n}\right] \tilde{\phi}_{n}+} \\
& \frac{\gamma}{2}\left[1-\tilde{f}_{n}^{2}(-h)\right]_{\tilde{\phi}_{n}}=\tilde{f}_{n}(-h) e^{-\frac{\gamma z}{2}} h_{t} \quad \text { (Eq. 1) }
\end{aligned}
$$

The linearized compressible flow solver reports later arrival due to increase in reduction term $\left(1-0.25 \mathrm{M}^{2}\right)$, where $M$ is a Mach number based on the ratio of surface long wave speed to sound speed.

\section{BOUSSINESQ MODEL}

We derive a Boussinesq model in spherical coordinates, which takes compressibility into account, with Mach number $M$ serving as an additional small parameter. The derivation of conservative forms and numerical implementation follows the approach described in Kirby et al. (2013). Model equations are omitted here due to space considerations.

The models have been validated through comparison with the solution of the full three-dimensional weakly compressible wave problem in vertical transects (or $\mathrm{x}$ z plane) over either constant or variable depth.

\section{TOHOKU-OKI 2011 TSUNAMI}

The model equations have been used to reconstruct tsunami waves generated by Tohoku-oki 2011 event. The generation mechanism of the event is modeled as a combination of the space and time varying coseismic seafloor deformation caused by the earthquake followed by a submarine mass failure (Tappin et al. 2013). Model results comparison compared to those obtained from incompressible wave equations and field data recorded by the Deepocean Assessment and Reporting of Tsunamis (DART) network will be presented at the Conference.

\section{REFERENCES}

Sammarco, Cecioni, Bellotti, Abdolali (2013) Depthintegrated equation for large-scale modelling of lowfrequency hydroacoustic waves. Journal of Fluid Mechanics, 722, R6

Abdolali, A., Kirby, J. T. and Bellotti, G., 2015, Depthintegrated equation for hydro-acoustic waves with bottom damping, Journal of Fluid Mechanics, 766, R1 doi:10.1017/jfm.2015.37

Abdolali, Kirby (2017) Role of Compressibility on Tsunami Propagation, Journal of Geophysical Research: Ocean, 122. https://doi.org/10.1002/2017JC013054

Kirby, Shi, Tehranirad, Harris, Grilli (2013) Dispersive tsunami waves in the ocean: model equations and sensitivity to dispersion and Coriolis effects. Ocean Modelling, 62, 39-55

Tappin, Grilli, Harris, Geller, Masterlark, Kirby, Shi, Ma, Thingbaijam, Mai (2014). Did a submarine landslide contribute to the 2011 Tohoku tsunami? Marine Geology, 357, 344-361 